\title{
Monoclonal antibodies and Fc fragments for treating solid tumors
}

\author{
This article was published in the following Dove Press journal: \\ Biologics:Targets and Therapy \\ 4 January 2012 \\ Number of times this article has been viewed
}

\section{Andrea M Eisenbeis \\ Stefan J Grau}

Department of Neurosurgery, University Hospital of Cologne, Cologne, Germany
Correspondence: Stefan Grau Department of Neurosurgery, University Hospital of Cologne, Kerpener Str. 62, 50937 Cologne, Germany Email stefan.grau@uk-koeln.de
Abstract: Advances in biotechnology, better understanding of pathophysiological processes, as well as the identification of an increasing number of molecular markers have facilitated the use of monoclonal antibodies and Fc fragments in various fields in medicine. In this context, a rapidly growing number of these substances have also emerged in the field of oncology. This review will summarize the currently approved monoclonal antibodies used for the treatment of solid tumors with a focus on their clinical application, biological background, and currently ongoing trials.

Keywords: targeted therapy, monoclonal antibodies, cancer, biological therapy

\section{Introduction}

Since the first description of monoclonal antibodies (mAbs) by Kohler and Milstein in 1975, ${ }^{1}$ numerous advances in molecular biology and biotechnology have streamlined the identification, genetic manipulation, and recombinant production of such antibodies. Over the past decade the use of antibodies has spread into different fields in medicine, such as autoimmune diseases, hematological disorders, cardiovascular diseases, infectious diseases, and diagnostics. ${ }^{2-4}$ Also, in the treatment of solid human tumors, biological therapeutics have emerged and gained importance for a growing number of cancer entities.

In the treatment of solid human tumors, biological therapeutics have also emerged and gained importance for a growing number of cancer forms. In contrast to conventional therapeutics such as chemotherapeutics or radiation, which both impose a high burden on nontumor tissue, the use of biological therapeutics aims at more specific, targeted therapy.

The concept of targeted therapy was facilitated by greater understanding of the biological pathways involved in the pathogenesis of cancer, and subsequently identification of cell surface antigens and bioactive molecules. ${ }^{5-7}$ A rather specific, and thus restrictive, expression pattern of these antigens and molecules in human tissue enables locally-focused targeting of antibodies to tumor tissue itself, as well as to nonneoplastic cells and substances relevant for tumor biology.

The introduction of mAbs in tumor therapy had a significant impact on pathological processes, particularly malignancies of solid and hematological origin. In particular, the use of mAbs has been established as mono, combination, first- and second-line, as well as palliative therapies. Their selectivity and specificity, unique pharmacokinetics, and ability to engage and activate the immune system differentiate these biologics from other small molecule anticancer drugs. 
This review summarizes the currently established biological therapeutics for treating solid tumors, with emphasis on the mAbs that have been approved by the United States Food and Drug Administration (FDA) for clinical use, as well as ongoing phase III studies and current $\mathrm{Fc}$ fusion proteins as of June 2011. Furthermore, the focus is on the targeted proteins and observed treatment effects associated with each antibody, the registration trials for their approved indications, and new emerging indications.

\section{Structure and nomenclature}

Biologically targeted therapy with mAbs has become an attractive treatment strategy for both solid tumors and hematological malignancies. The hybridoma technology can produce antibodies that are products of single antibodyproducing cells, and thus have a single variable region associated with one constant region, in other words mAbs. ${ }^{1}$

The first therapeutic mAbs were derived from immunizing mice against epidermal growth factor receptor (EGFR) proteins. These murine mAbs (suffix “-omab") were associated with activation of the immune system, and development of antimurine antibody responses that induced severe anaphylaxis, which decreased the circulating half-life and finally led to reduced efficacy., As a consequence, chimerized ("-ximab") antibodies were generated by genetically replacing the human constant sequence with a variable murine domain with antigenic activity. The production of fully humanized mAbs (“-zumab"), replacing murine residues in complementarity determining regions with human sequences, finally reduced immunogenicity and improved their efficacy and safety for use in humans. ${ }^{10}$ These technological advances resulted in molecules with a long circulating half-life that, depending on the Fc selection, interact with human complement and/or effector cells to produce cell-mediated cytotoxicity or antibody dependent cell-mediated cytotoxicity (ADCC).

The mechanisms of action may be explained by an induction and concentration of host defense mechanisms, the neutralization of biologically active substances, and/or impairment of specific intracellular pathways. These effects may result in target-oriented immune response, reduced tumor proliferation itself, and/or increased efficacy of radio- or chemotherapy.

\section{Limitations and side effects}

While mAb-based therapies offer novel strategies to target cytotoxicity exclusively to malignant cells, successful application with maximal efficacy is often limited by common obstacles. The therapeutic benefit of mAbs may be limited by low or heterogeneous expression of target antigens by tumor cells, high background expression of antigens by tumor cells, and host antibody immune responses to mAb binding. These problems may be aggravated by, for example, compensatory responses of affected cells to $\mathrm{mAb}$ treatment in terms of an upregulation of alternative or primary redundant signaling molecules. This phenomenon may represent one escape mechanism resulting in secondary tumor regrowth and/or increasing angiogenesis.

Additionally, physical obstructions for antibody binding such as endothelial, interstitial, and epithelial barriers complicate the delivery of mAbs to target cells and thus reduce therapeutic efficacy. ${ }^{11}$ To overcome this obstacle, antibody fragments lacking the Fc region have been developed. The smaller molecule size results in improved tissue permeability, however, it also leads to reduced plasma half-life due to increased renal elimination. Furthermore, the lack of an $\mathrm{Fc}$ region excludes a direct activation of the complement system and consequently direct cytotoxic activity.

A wide range of side effects of mAb therapy has been reported, which may be caused by antibody binding to nonneoplastic tissue, thus interfering with physiological processes and inducing autoimmune responses. Furthermore, the desired immune response with a resulting secretion of proinflammatory cytokines may induce severe symptoms as fever, malaise, and headaches.

\section{mAbs}

\section{Current clinical applications for solid tumors}

The following antibodies in clinical use are summarized in Table 1.

\section{Trastuzumab (Herceptin ${ }^{\circledR}$ )}

Trastuzumab is a humanized $\mathrm{mAb}$ that recognizes an epitope on the extracellular domain of the human EGFR-2 (HER2/ neu, c-erbB2). ${ }^{12}$ Several studies reported that amplification of the erbB2 proto-oncogene was associated with overexpression of HER 2 in tumor cells in approximately $25 \%$ of breast cancers, adenocarcinomas of the ovary, prostate, lung, and gastrointestinal tract. ${ }^{13,14}$

To prolong the systemic half-life of trastuzumab the idiotopes from the hypervariable region that bind HER2 with high affinity and avidity were linked with elements of human immunoglobulin (Ig) G1 antibody. Due to the human IgG1 constant regions of the humanized $\mathrm{mAb}$, trastuzumab is very effective for inducing ADCC against breast cancer cells that overexpress HER2 in vitro but is ineffective for inducing cell-mediated cytotoxicity. Trastuzumab was granted regulatory approval in 1999 as monotherapy for metastatic 
Table I Food and Drug Administration approved monoclonal antibodies for therapy of solid tumors

\begin{tabular}{|c|c|c|c|}
\hline Antibody & Trade name & Target & Indication \\
\hline Cetuximab & Erbitux $^{\circledR}$ & EGFR & $\begin{array}{l}\text { CRC (m), SCCHN } \\
\text { (loc ad, rec) }\end{array}$ \\
\hline Panitumumab & Vectibix $^{\circledR}$ & EGFR & $\mathrm{CRC}(\mathrm{m})$ \\
\hline Trastuzumab & Herceptin $^{\circledR}$ & HER2 & $\begin{array}{l}B C(a d, m), A C \text { of } \\
\text { GE junction }\end{array}$ \\
\hline Bevacizumab & Avastin $^{\circledR}$ & VEGF & $\begin{array}{l}\text { CRC (m), SCCHN } \\
\text { (loc ad, rec), BC } \\
\text { (m), RCC (ad), } \\
\text { glioblastoma }\end{array}$ \\
\hline Girentuximab & Rencarex $^{\circledR}$ & $\begin{array}{l}\text { Carbonic } \\
\text { anhydrase IX }\end{array}$ & $\mathrm{RCC}$ \\
\hline Ipilimumab & Yervoy $^{\circledR}$ & CTLA-4 & MM (met) \\
\hline
\end{tabular}

Abbreviations: AC, adenocarcinoma; ad, advanced; BC, breast cancer; CRC colorectal cancer; CTLA-4, cytotoxic T lymphocyte antigen-4; EGFR, epidermal growth factor receptor; GE, gastroesophageal; HER2, human epidermal growth factor receptor-2; loc adv, locally advanced; m, metastatic; MM, malignant melanoma; RCC, renal cell carcinoma; rec, recurrent; SCCHN, squamous cell carcinoma of the head and neck; VEGF, vascular endothelial growth factor.

breast cancer and thus became the first mAb approved based on activity in a solid tumor. ${ }^{2}$

In two large trials with HER2-overexpressing breast cancer patients having had little or no prior chemotherapy, the combination of chemotherapy with trastuzumab led to a higher objective response rate, a longer time to progression, longer duration of response, and greater overall survival compared to chemotherapy alone. ${ }^{15}$

In 2006, trastuzumab became the only tyrosine kinasetargeted agent approved for the adjuvant treatment of solid tumors, based on pooled data from $>3000$ node-positive, or high-risk node-negative patients from two clinical trials. ${ }^{16}$

Since 2008, trastuzumab has been released as a single agent in second-line therapy in metastatic breast cancer. The most severe toxicities associated with HER2/neu-targeting antibodies are cardiomyopathy and pulmonary disorders. ${ }^{17}$

\section{Bevacizumab (Avastin ${ }^{\circledR}$ )}

Bevacizumab is a humanized $\operatorname{IgG1} \mathrm{mAb}$ that blocks binding of vascular endothelial growth factor (VEGF) to its receptors VEGFR1 and VEGFR2 on vascular endothelium. VEGF is regarded as the key regulator of tumor-associated angiogenesis, and is produced by most types of malignant cells. While the initial hypothesis that antiangiogenic agents lead to starvation of tumor tissue has been revised in favor of a structural reorganization of tumor vessels during antiangiogenic treatment, increased responsiveness to radiation and chemotherapy in combination with antiVEGF agents has been reported.

Bevacizumab was originally approved in 2004 as the first VEGF inhibitor for first-line treatment of metastatic colorectal cancer (mCRC) after promising results in a key trial. ${ }^{18}$ In this phase III trial involving 813 patients with mCRC, 402 patients received irinotecan, fluorouracil, and leucovorin (IFL) plus bevacizumab, while 411 received IFL plus placebo. Overall survival significantly increased from 15.6 months in the control group (IFL plus placebo) to 20.3 months in the IFL/bevacizumab group.

Based on a randomized, multicenter trial that proved longer overall survival and overall response rate with bevacizumab/oxaliplatin, fluorouracil, and leucovorin compared to oxaliplatin, fluorouracil, and leucovorin monotherapy in patients with mCRC, bevacizumab was approved as a second-line agent for this indication. ${ }^{19}$

Subsequently, bevacizumab was approved for first-line combination treatment of advanced and metastatic nonsmall cell lung cancer in 2006 and for combination therapy with paclitaxel in metastatic breast cancer in 2008 by the FDA..$^{20,21}$

Final results of the phase III study (AVOREN) conducted in patients with metastatic renal cell carcinoma who had undergone nephrectomy showed a median progression-free survival of 10.2 months for the bevacizumab plus interferon arm compared to 5.4 months for the interferon and placebo arm. ${ }^{22}$

In 2009, the FDA approved bevacizumab as a secondline treatment for patients with recurrent glioblastoma after prior standard therapy. ${ }^{23}$ This approval was not given by the European Medicines Agency due to the weakness of the published data. Currently, a multicentric phase III trial is underway for glioblastoma (AVAGLIO).

Due to the widespread expression of VEGF, a large range of side effects has been reported. Among those, the most frequent are hypertension, skin changes, abdominal pain, and general weakness. . $^{18,21,23,24}$

\section{Cetuximab (Erbitux $\left.{ }^{\circledR}\right)$ and panitumumab (Vectibix ${ }^{\circledR}$ )}

Observed overexpression of EGFR in colorectal carcinomas led to the development of antiEGFR mAb such as cetuximab and panitumumab that bind to EGFRs and competitively block ligand binding. As a consequence, blocking phosphorylation and activation of several tyrosine kinases intercepts signal transduction, resulting in inhibition of tumor growth and induction of apoptosis in vitro. ${ }^{25}$

Cetuximab, a chimeric monoclonal IgG1 antibody, develops its antitumor efficacy through specific targeting of EGFRs, inducing receptor internalization and degradation with downregulation of EGFR expression. The therapeutic mechanism is mainly the induction of ADCC rather than immunotherapeutical effects. ${ }^{26}$

Cetuximab is predominantly implemented as an additional agent to chemo- and radiotherapy in $\mathrm{mCRC}$, as well as head 
and neck cancer. It was first approved for the treatment of refractory $\mathrm{mCRC}$ in combination with irinotecan in patients who had EGFR-detectable, irinotecan-refractory mCRC. ${ }^{27}$

Following a continuative study that showed a reduced risk for disease progress of $\mathrm{mCRC}$ using cetuximab in combination with irinotecan, folinic acid, and 5-fluorouracil, cetuximab was approved as a first-line treatment for CRC in $2009 .{ }^{28}$

It also gained FDA approval for combination with radiotherapy to treat locally advanced squamous cell carcinoma of the head and neck (SCCHN), ${ }^{29,30}$ as well as second-line monotherapy in recurrent metastatic SCCHN. ${ }^{31}$

One randomized, open-label ongoing phase III multicenter study (NCT01309126) is evaluating overall survival of patients with wild-type $k R A S C R C$ after treatment with a combination of cetuximab and Imprime $\mathrm{PGG}^{\circledR}$ or cetuximab alone. The study started in April 2011 and will be completed in September 2012 after an estimated enrollment of 795 patients.

Serious cetuximab-related toxicities include hypersensitivity, infusion-related reactions, and interstitial lung disease including pulmonary hemorrhage. ${ }^{32}$

Panitumumab is a fully humanized IgG2 antibody that like cetuximab interacts with the EGF-1 receptor, impairing signal transduction and subsequently leading to less cell proliferation and resistance to apoptosis. However, panitumumab does not induce EGFR degradation.

Panitumumab efficacy was evaluated in four phase III trials. ${ }^{24,33-35}$ It was granted regulatory approval as the first fully human $\mathrm{mAb}$ based on a randomized phase III trial ${ }^{33}$ where 463 patients with $\mathrm{mCRC}$ who had received both panitumumab and best supportive care (BSC) showed an improvement in their overall response rate and progressionfree survival compared to treatment with BSC alone. Response rates in the combined therapy group were even higher in patients positive for wild-type $k R A S$ (an intracellular molecule involved in EGF signaling) whereas these drugs had no effect in patients with mutated $k R A S{ }^{36}$ There was no proof of benefit for combination of panitumumab with chemotherapy in first-line treatment of mCRC. ${ }^{37}$

The most commonly reported adverse effects of antiEGFR $\mathrm{mAbs}$ are explained by the widespread expression of EGFR on various tissues. These include acneiform rash, malaise, hypomagnesemia, pneumonia, and headaches. Compared to cetuximab, fewer common adverse events, especially dermatological toxicity, accompanied treatment with panitumumab. ${ }^{38}$

In conclusion, cetuximab and panitumumab are currently part of standard therapy for $\mathrm{mCRC}$, although results from ongoing trials might elucidate further treatment indications.

\section{Ipilimumab (Yervoy $\left.{ }^{\circledR}\right)$}

Ipilimumab, a novel human IgG1 mAb inhibiting the cytotoxic T lymphocyte antigen-4, has been evaluated for treating metastatic melanoma. FDA granted priority review for ipilimumab in March 2011 as the first agent indicated for unresectable, metastatic, or untreated stage III or IV melanoma in adults.

Recently, results of a phase III randomized, double-blind trial were published demonstrating an overall survival benefit for patients with metastatic melanoma who were treated with ipilimumab as monotherapy or with the glycoprotein 100 vaccine. ${ }^{39}$ The trial did not show the suggested synergistic effect of glycoprotein 100, nor that the efficacy of ipilimumab was altered by the immune response. Thus, the FDA approved ipilimumab as monotherapy in unresectable, metastatic, or untreated stage III or IV melanoma in adults.

Cytotoxic T lymphocyte antigen-4-inhibiting mAbs may induce autoimmunity, enterocolitis, and hypopituitarism. ${ }^{17}$

\section{Novel mAbs currently being investigated in phase III trials Girentuximab (Rencarex ${ }^{\circledR}$ )}

Girentuximab is an IgG1 kappa light-chain antibody that binds to carbonic anhydrase IX (G250-antigen), a cell surface antigen expressed in $95 \%$ of clear cell renal cell carcinoma. The proposed mechanism of action is increased stimulation of natural killer cells with activity focused on tumor cells via ADCC.

In phase I/II studies, the combination of girentuximab with low-dose interferon-alpha in patients with progressive metastatic renal cell carcinoma demonstrated clinical benefit, a good response rate, and significant prolongation of the median survival time $(30 \%)$ in more than 130 treated patients. $^{40}$

For patients with primary clear cell renal cell carcinoma, a phase III trial (NCT00087022) using girentuximab is currently in progress and will end in 2013.

To date, no serious adverse event related to the study medication has been published described for Rencarex in the clinical phase I/II trials. Adverse events mainly compromised fever, flu-like syndromes, and headache. ${ }^{40}$

\section{Zalutumumab}

Zalutumumab, a human IgG1 mAb that targets EGFR, is undergoing evaluation in an open-label, randomized phase III study (NCT00382031) for treating patients with incurable SCCHN who have failed standard platinum-based chemotherapy. Previous studies combining zalutumumab and 
BSC, compared to only BSC, resulted in improved median overall survival from 5.2 to 6.7 months. ${ }^{41}$

Another ongoing phase III study (primary completion date: January 2012; NCT00496652) aims to determine whether addition of the fully human EGFR antibody zalutumumab to primary curative radiotherapy increases locoregional control in SCCHN. Enrollment is around 600 patients and the estimated study completion date is September 2016.

As mentioned above, the expression pattern of EGFR may lead to adverse effects which might be similar to that of other EGFR antibodies.

\section{Tremelimumab}

Tremelimumab is a fully human anticytotoxic $\mathrm{T}$ lymphocyte antigen-4 IgG2 antibody that modulates the immunological response using the same mechanism as ipilimumab. ${ }^{42}$ The advantage of tremelimumab over ipilimumab is a significantly longer half-life.

Tremelimumab failed to show a significant improvement in overall survival versus chemotherapy alone in a phase III trial for surgically incurable metastatic melanoma patients without prior chemotherapy for metastatic disease. ${ }^{43}$ The study was discontinued after the interim analysis of tremelimumab compared to chemotherapy with dacarbazine or temozolomide (11.8 months to 10.7 months).${ }^{44}$ One ongoing trial studying the side effects and best dose of tremelimumab together with a CD40 agonist mAb CP-870 in 893 patients with metastatic melanoma has an estimated study completion date in March 2013 (NCT01103635).

In a phase II trial of tremelimumab a large proportion of patients $(77 \%)$ developed treatment-related adverse events, with $19 \%$ showing serious adverse events including endocrine dysfunctions and colitis. ${ }^{44}$

\section{Necitumumab}

Necitumumab (IMC-11F8) is an EGFR-targeting IgG1 mAb which is currently being evaluated in two phase III trials for treating nonsquamous stage IIIB or IV nonsmall cell lung cancer. An open-labeled, randomized, active-controlled study (NCT00982111) is comparing the effect of necitumumab in combination with standard chemotherapy with standard chemotherapy alone. Data will be collected from 947 estimated patients until October 2011 with an estimated study completion date in May 2012.

\section{Farletuzumab}

Farletuzumab is a humanized $\operatorname{IgG} 1 \mathrm{mAb}$ that targets human folate receptor alpha, which is expressed in most epithelial ovarian cancers. Farletuzumab administered as a monotherapeutic agent or in combination with carboplatin and taxane in patients with platinum-sensitive relapsed epithelial ovarian cancers showed normalization of the biomarker CA125 as an indirect sign of disease response.

A current phase III study (NCT00849667) in initially platinum-sensitive relapsed ovarian cancer patients is comparing farletuzumab, combined with carboplatin and taxane, to chemotherapy alone. The estimated study completion date, with 900 patients, is September 2012. ${ }^{45}$

Another randomized, double-blind phase III trial (NCT00738699) is being conducted to find out whether farletuzumab increases the efficacy and safety of paclitaxel in refractory relapsed ovarian cancer. In addition, the safety of farletuzumab, carboplatin, and pegylated liposomal doxorubicin combination in patients with platinum-sensitive ovarian cancer is being assessed in an ongoing nonrandomized phase III trial (NCT01004380).

\section{Trastuzumab emtansine}

Trastuzumab emtansine is an immunoconjugate formed by conjugation of trastuzumab to cytotoxic maytansinoid DM1 that targets HER2.

A current randomized, multicenter, two-arm, open-label study is evaluating the efficacy and safety of trastuzumab emtansine in comparison to treatment of the physician's choice in patients with metastatic or nonresectable locally advanced/recurrent HER2-positive breast cancer who have received at least two prior regimens of HER2-directed therapy. The estimated completion date for the primary outcome measure is April 2015.

Data from two additional phase III studies will compare trastuzumab emtansine to the combination of capecitabine and lapatinib in patients with HER2-positive, locally advanced, or metastatic breast cancer in adult patients who have received prior treatment with trastuzumab (NCT00829166). The estimated primary completion date for 580 enrolled patients is August 2013.

One randomized, three-arm, multicenter study (NCT01120184) is evaluating the efficacy and safety of trastuzumab emtansine with pertuzumab, or trastuzumab emtansine with placebo, versus the combination of trastuzumab plus taxane (docetaxel or paclitaxel) in patients with HER2-positive breast cancer. This started in July 2010, and the estimated primary completion date is July 2017.

\section{Pertuzumab}

Pertuzumab is a humanized antiHER2 IgG1, and the first of its kind in a group of agents inhibiting HER dimerization by 
binding to the dimerization domain of HER2. The resulting inhibition of HER2 forming a complex with other HER receptors may result in slowed tumor growth. ${ }^{46}$

Currently, three phase III studies are evaluating single treatment by pertuzumab or combination therapy in previously untreated HER2 positive metastatic breast cancer patients. A phase III study (NCT01120184) is examining the combination of pertuzumab and trastuzumab emtansine. Another phase III study (NCT00567190) is evaluating the safety and efficacy of pertuzumab, trastuzumab, and docetaxel compared to trastuzumab, docetaxel, and a placebo. The estimated primary completion date is March 2012.

The purpose of another ongoing randomized, doubleblind phase III study (NCT01358877) is to assess pertuzumab in addition to chemotherapy plus trastuzumab as an adjuvant treatment therapy in patients with operable HER2positive primary breast cancer. After surgery, patients are randomized to pertuzumab or a placebo. Starting in October 2011, the estimated primary completion date is July 2024.

\section{Ramucirumab}

Ramucirumab is a human IgG1 mAb that, similar to bevacizumab, targets the VEGF/VEGFR system and consequently tumor angiogenesis. Unlike bevacizumab, which binds VEGF, ramucirumab binds to VEGFR2. It is being currently evaluated in six phase III trials.

A randomized, double-blinded study is comparing ramucirumab and $\mathrm{BSC}$ versus a placebo and $\mathrm{BSC}$ in patients with adenocarcinomas of the gastroesophageal junction (NCT00917384). Starting in October 2009, with an estimated enrollment of 315 patients, the predicted primary completion date is May 2012.

A second ongoing phase III randomized, multicenter double-blind, placebo-controlled trial (NCT01170663) with 663 enrolled patients is comparing paclitaxel with or without ramucirumab in metastatic gastric adenocarcinoma after first-line treatment. The final data collection date for the primary outcome measure is May 2013. Further ongoing phase III trials include treatment options with addition of ramucirumab to irinotecan, folinic acid, and 5-fluorouracil chemotherapy in second-line mCRC (NCT01183780) or administration of ramucirumab versus a placebo as second-line treatment in patients with hepatocellular carcinoma following first-line therapy with sorafenib (NCT01140347, estimated study completion date: December 2012).
The combination of ramucirumab with docetaxel versus docetaxel plus a placebo in the treatment of stage IV nonsmall cell lung cancer is currently being evaluated in a randomized, double-blind phase III study (NCT01168973). The estimated patient enrollment is 1242 , and the study completion date is expected in June 2014.

The effects of docetaxel and ramucirumab for previously untreated patients with HER2-negative breast cancer are being evaluated in a multicenter, multinational, randomized, double-blind, placebo-controlled phase III study (NCT00703326). The estimated enrollment is 1113 patients and the predicted completion date is August 2015.

\section{Catumaxomab $\left(\operatorname{Removab}^{\circledR}\right)$}

Catumaxomab is a trifunctional antibody with two binding sites and a functional Fc domain. It targets the epithelial cell adhesion molecule as well as the CD3 receptor on T lymphocytes. ${ }^{47}$

This novel antibody class has the capability to redirect T cells and accessory cells, eg, macrophages, dendritic cells, and natural killer cells, to the tumor site after its activation by regulated intramembrane proteolysis. ${ }^{48-50}$

In a two-arm, randomized, open-label phase II/III study (NCT00836654) in patients with malignant and symptomatic ascites due to epithelial tumors, a reduction of necessary paracentesis was observed for the treatment arm. However, no significant increase of overall survival was reported.

\section{Nimotuzumab (TheraCIM ${ }^{\circledR}$, Theraloc ${ }^{\circledR}$ )}

Nimotuzumab is a recombinant humanized murine $\operatorname{IgG} 1$ $\mathrm{mAb}$ that blocks both EGF and transforming growth factor. Compared to other EGFR-targeting antibodies, nimotuzumab has a slightly lower binding affinity, which is thought to account for an improved side-effect profile.

Several clinical trials are in progress globally to evaluate nimotuzumab in different indications of solid tumors. The combination of nimotuzumab and concurrent chemoradiotherapy has been approved in several countries for use in SCCHN, and is currently being studied in end-stage phase II and phase III trials for treating locally advanced esophageal cancer (NCT01249352, NCT01402180).

Another ongoing study is evaluating standard radiotherapy plus concomitant and adjuvant nimotuzumab plus temozolomide versus standard radiotherapy plus concomitant and adjuvant temozolomide in patients with newly diagnosed glioblastoma multiforma (NCT00753246). The study started in 2007 and the estimated completion date is August 2011. 


\section{Fc fragments Aflibercept}

The aflibercept molecule was originally synthesized as a fusion protein combining the constant region $(\mathrm{Fc})$ of $\mathrm{IgG} 1$ with the first three domains of VEGFR1. It was found to maintain impressive picomolar binding affinity to VEGF, and was thus called a VEGF-trap.

Potential treatment effects are currently being seen in ongoing phase III studies in patients with mCRC (NCT00561470), and as second-line treatment of patients with locally advanced or metastatic nonsmall cell lung cancer (NCT00532155). In patients with metastatic androgenindependent prostate cancer, a randomized double-blind phase III trial showed no improved survival after treatment with aflibercept added to gemcitabine in a planned interim analysis (NCT00519285).

\section{AMG-386}

AMG 386, used as a competitive inhibitor of angiogenesis, comprises a human IgG1 Fc fragment and peptide specificity towards the angiopoietins Ang-1 and Ang-2.

A placebo-controlled phase III study to determine whether treatment of paclitaxel plus AMG 386 is efficient in women with epithelial ovarian cancer, primary peritoneal cancer, or fallopian tube cancer (NCT01204749) will be completed in April 2017.

\section{Summary}

Advances in molecular biology and biotechnology have led to a new class of therapeutics, mAbs, designed to treat an increasing number of indications. The clear advantages of mAbs for treating solid tumors lies in a more tumor-specific, and even cell-specific approach, which can reduce adverse effects frequently observed during conventional therapies. However, despite the currently exploding number of new agents, mAbs as well as Fc fragments are far from being a magic bullet in tumor therapy, since they still exhibit their own treatment-related risks as well as clear limitations. This explains the increasing focus on developing more specific, fine-tuned, and personalized approaches to use these agents as a powerful device in oncological therapy. In the future, not only a growing number of relevant antigens may be discovered, but also combination with other approaches (eg, linking antibodies to radionuclides, immunostimulants, or toxins) may allow better tumor control and increase survival in a variety of cancers.

Due to the large body of primary literature about $\mathrm{mAb}$ therapy in solid tumors, only selected references are given, and results from recent publications and articles that were relevant to phase II and III studies are emphasized.

\section{Disclosure}

The authors report no conflicts of interest in this work.

\section{References}

1. Kohler G, Milstein C. Continuous cultures of fused cells secreting antibody of predefined specificity. Nature. 1975;256(5517):495-497.

2. Dillman RO. Perceptions of Herceptin: a monoclonal antibody for the treatment of breast cancer. Cancer Biother Radiopharm. 1999;14(1):5-10.

3. Dillman RO. Monoclonal antibodies for treating cancer. Ann Intern Med. 1989;111(7):592-603.

4. Dillman RO. Antibodies as cytotoxic therapy. J Clin Oncol. 1994; 12(7):1497-1515.

5. Wigzell H. The immune system as a therapeutic agent. Sci Am. 1993; 269(3):126-134

6. Boon T. Teaching the immune system to fight cancer. Sci Am. 1993; 268(3):82-89.

7. Jiang H, Chess L. Regulation of immune responses by T cells. $N$ Engl J Med. 2006;354(11):1166-1176.

8. Abramowicz D, Crusiaux A, Goldman M. Anaphylactic shock after retreatment with OKT3 monoclonal antibody. N Engl J Med. 1992; 327(10):736.

9. Bajorin DF, Chapman PB, Wong GY, et al. Treatment with high dose mouse monoclonal (anti-GD3) antibody R24 in patients with metastatic melanoma. Melanoma Res. 1992;2(5-6):355-362.

10. Cobleigh MA, Vogel CL, Tripathy D, et al. Multinational study of the efficacy and safety of humanized anti-HER2 monoclonal antibody in women who have HER2-overexpressing metastatic breast cancer that has progressed after chemotherapy for metastatic disease. J Clin Oncol. 1999;17(9):2639-2648.

11. Christiansen J, Rajasekaran AK. Biological impediments to monoclonal antibody-based cancer immunotherapy. Mol Cancer Ther. 2004;3(11): 1493-1501.

12. Baselga J, Tripathy D, Mendelsohn J, et al. Phase II study of weekly intravenous trastuzumab (Herceptin) in patients with HER2/ neu-overexpressing metastatic breast cancer. Semin Oncol. 1999; 26(4 Suppl 12):78-83.

13. Schechter AL, Stern DF, Vaidyanathan L, et al. The neu oncogene: an erb-B-related gene encoding a 185,000-Mr tumour antigen. Nature. 1984;312(5994):513-516.

14. Slamon DJ, Clark GM, Wong SG, Levin WJ, Ullrich A, McGuire WL. Human breast cancer: correlation of relapse and survival with amplification of the HER-2/neu oncogene. Science. 1987;235(4785):177-182.

15. Slamon DJ, Leyland-Jones B, Shak S, et al. Use of chemotherapy plus a monoclonal antibody against HER2 for metastatic breast cancer that overexpresses HER2. N Engl J Med. 2001;344(11):783-792.

16. Romond EH, Perez EA, Bryant J, et al. Trastuzumab plus adjuvant chemotherapy for operable HER2-positive breast cancer. NEngl J Med. 2005;353(16):1673-1684.

17. Heimann DM, Weiner LM. Monoclonal antibodies in therapy of solid tumors. Surg Oncol Clin N Am. 2007;16(4):775-792, viii.

18. Hurwitz H, Fehrenbacher L, Novotny W, et al. Bevacizumab plus irinotecan, fluorouracil, and leucovorin for metastatic colorectal cancer. N Engl J Med. 2004;350(23):2335-2342.

19. Giantonio BJ, Catalano PJ, Meropol NJ, et al. Bevacizumab in combination with oxaliplatin, fluorouracil, and leucovorin (FOLFOX4) for previously treated metastatic colorectal cancer: results from the Eastern Cooperative Oncology Group Study E3200. J Clin Oncol. 2007;25(12):1539-1544.

20. Sandler A, Gray R, Perry MC, et al. Paclitaxel-carboplatin alone or with bevacizumab for non-small-cell lung cancer. N Engl J Med. 2006; 355(24):2542-2550. 
21. Miller K, Wang M, Gralow J, et al. Paclitaxel plus bevacizumab versus paclitaxel alone for metastatic breast cancer. $N$ Engl J Med. 2007; 357(26):2666-2676

22. Escudier B, Pluzanska A, Koralewski P, et al. Bevacizumab plus interferon alfa-2a for treatment of metastatic renal cell carcinoma: a randomised, double-blind phase III trial. Lancet. 2007;370(9605): 2103-2111.

23. Friedman HS, Prados MD, Wen PY, et al. Bevacizumab alone and in combination with irinotecan in recurrent glioblastoma. J Clin Oncol. 2009;27(28):4733-4740.

24. Hecht JR, Mitchell E, Chidiac T, et al. A randomized phase IIIB trial of chemotherapy, bevacizumab, and panitumumab compared with chemotherapy and bevacizumab alone for metastatic colorectal cancer. J Clin Oncol. 2009;27(5):672-680.

25. Huang SM, Bock JM, Harari PM. Epidermal growth factor receptor blockade with $\mathrm{C} 225$ modulates proliferation, apoptosis, and radiosensitivity in squamous cell carcinomas of the head and neck. Cancer Res. 1999;59(8):1935-1940.

26. Vincenzi B, Zoccoli A, Pantano F, Venditti O, Galluzzo S. Cetuximab: from bench to bedside. Curr Cancer Drug Targets. 2010;10(1):80-95.

27. Cunningham D, Humblet Y, Siena S, et al. Cetuximab monotherapy and cetuximab plus irinotecan in irinotecan-refractory metastatic colorectal cancer. $N$ Engl J Med. 2004;351(4):337-345.

28. Van Cutsem E, Kohne CH, Hitre E, et al. Cetuximab and chemotherapy as initial treatment for metastatic colorectal cancer. NEngl J Med. 2009; 360(14):1408-1417.

29. Bonner JA, Harari PM, Giralt J, et al. Radiotherapy plus cetuximab for squamous-cell carcinoma of the head and neck. N Engl J Med. 2006; 354(6):567-578.

30. Bonner JA, Harari PM, Giralt J, et al. Radiotherapy plus cetuximab for locoregionally advanced head and neck cancer: 5-year survival data from a phase 3 randomised trial, and relation between cetuximab-induced rash and survival. Lancet Oncol. 2010;11(1):21-28.

31. Vermorken JB, Trigo J, Hitt R, et al. Open-label, uncontrolled, multicenter phase II study to evaluate the efficacy and toxicity of cetuximab as a single agent in patients with recurrent and/or metastatic squamous cell carcinoma of the head and neck who failed to respond to platinumbased therapy. J Clin Oncol. 2007;25(16):2171-2177.

32. Harding J, Burtness B. Cetuximab: an epidermal growth factor receptor chemeric human-murine monoclonal antibody. Drugs Today (Barc). 2005;41(2):107-127.

33. Van Cutsem E, Peeters M, Siena S, et al. Open-label phase III trial of panitumumab plus best supportive care compared with best supportive care alone in patients with chemotherapy-refractory metastatic colorectal cancer. J Clin Oncol. 2007;25(13):1658-1664.

34. Siena S, Peeters M, Van Cutsem E, et al. Association of progressionfree survival with patient-reported outcomes and survival: results from a randomised phase 3 trial of panitumumab. Br J Cancer. 2007;97(11): 1469-1474.

35. Peeters M, Price TJ, Cervantes A, et al. Randomized phase III study of panitumumab with fluorouracil, leucovorin, and irinotecan (FOLFIRI) compared with FOLFIRI alone as second-line treatment in patients with metastatic colorectal cancer. J Clin Oncol. 2010;28(31):4706-4713.
36. Amado RG, Wolf M, Peeters M, et al. Wild-type KRAS is required for panitumumab efficacy in patients with metastatic colorectal cancer. J Clin Oncol. 2008;26(10):1626-1634.

37. Wirth LJ, Allen AM, Posner MR, et al. Phase I dose-finding study of paclitaxel with panitumumab, carboplatin and intensity-modulated radiotherapy in patients with locally advanced squamous cell cancer of the head and neck. Ann Oncol. 2010;21(2):342-347.

38. Lacouture ME, Mitchell EP, Piperdi B, et al. Skin toxicity evaluation protocol with panitumumab (STEPP), a phase II, open-label, randomized trial evaluating the impact of a pre-emptive skin treatment regimen on skin toxicities and quality of life in patients with metastatic colorectal cancer. J Clin Oncol. 2010;28(8):1351-1357.

39. Hodi FS, O'Day SJ, McDermott DF, et al. Improved survival with ipilimumab in patients with metastatic melanoma. NEngl J Med. 2010; 363(8):711-723.

40. Siebels M, Rohrmann K, Oberneder R, et al. A clinical phase I/II trial with the monoclonal antibody cG250 (RENCAREX ${ }^{\circledR}$ ) and interferonalpha-2a in metastatic renal cell carcinoma patients. World J Urol. 2011; 29(1):121-126.

41. Machiels JP, Subramanian S, Ruzsa A, et al. Zalutumumab plus best supportive care versus best supportive care alone in patients with recurrent or metastatic squamous-cell carcinoma of the head and neck after failure of platinum-based chemotherapy: an open-label, randomised phase 3 trial. Lancet Oncol. 2011;12(4):333-343.

42. O’Day SJ, Hamid O, Urba WJ. Targeting cytotoxic T-lymphocyte antigen-4 (CTLA-4): a novel strategy for the treatment of melanoma and other malignancies. Cancer. 2007;110(12):2614-2627.

43. Ribas A, Hauschild A, Kefford A, et al. Phase III, open-label, randomized, comparative study of tremelimumab (CP-675,206) and chemotherapy (temozolomide [TMZ] or dacarbazine [DTIC]) in patients with advanced melanoma. J Clin Oncol. 2008;26(20 Suppl): Abstract LBA9011.

44. Kirkwood JM, Lorigan P, Hersey P, et al. Phase II trial of tremelimumab $(\mathrm{CP}-675,206)$ in patients with advanced refractory or relapsed melanoma. Clin Cancer Res. 2010;16(3):1042-1048.

45. White A, Coleman R, Armstrong D, et al. Efficacy and safety of farletuzumab, a humanized monoclonal antibody to folate receptor alpha, in platinum-sensitive, relapsed ovarian cancer subjects: final data from a multicenter phase II study. J Clin Oncol. 2010;28(15 Suppl): Abstract 5001.

46. Scheuer W, Friess T, Burtscher H, Bossenmaier B, Endl J, Hasmann M. Strongly enhanced antitumor activity of trastuzumab and pertuzumab combination treatment on HER2-positive human xenograft tumor models. Cancer Res. 2009;69(24):9330-9336.

47. Linke R, Klein A, Seimetz D. Catumaxomab: clinical development and future directions. MAbs. 2010;2(2):129-136.

48. Maetzel D, Denzel S, Mack B, et al. Nuclear signalling by tumourassociated antigen EpCAM. Nat Cell Biol. 2009;11(2):162-171.

49. Munz M, Baeuerle PA, Gires O. The emerging role of EpCAM in cancer and stem cell signaling. Cancer Res. 2009;69(14):5627-5629.

50. Carpenter G, Red BM. EpCAM: another surface-to-nucleus missile. Cancer Cell. 2009;15(3):165-166.

\section{Publish your work in this journal}

Biologics: Targets \& Therapy is an international, peer-reviewed journal focusing on the patho-physiological rationale for and clinical application of Biologic agents in the management of autoimmune diseases, cancers or other pathologies where a molecular target can be identified. This journal is indexed on PubMed Central, CAS, EMBase, Scopus

\section{Dovepress}

and the Elsevier Bibliographic databases. The manuscript management system is completely online and includes a very quick and fair peerreview system, which is all easy to use. Visit http://www.dovepress. com/testimonials.php to read real quotes from published authors. 